\title{
FUNCTIONAL INSIGHTS AND MOLECULAR MODEL ANALYSES OF Thermotoga maritima XYLANASES REVEAL THERMOSTABILITY AND COMPLEX EVOLUTIONARY LINEAGE
}

\author{
Aqsa Parvaiz ${ }^{1}$, Muhammad Rizwan Javed ${ }^{2}$, Ghulam Mustafa', Muhammad Aamer Mehmood², \\ Muhammad Tahir Ul Qamar ${ }^{3}$, Ayesha Pervaiz ${ }^{4}$ and Faiz Ahmad Joyia,"*
}

\author{
${ }^{1}$ Center of Agricultural Biochemistry and Biotechnology (CABB), University of Agriculture, Faisalabad-38040, \\ Pakistan; ${ }^{2}$ Department of Bioinformatics and Biotechnology, Government College University, Faisalabad-38000, \\ Pakistan; ${ }^{3}$ College of Informatics, Huazhong Agricultural University, Wuhan-430070, P.R. China; ${ }^{4}$ Institute of \\ Pharmacy, Physiology and Pharmacology, University of Agriculture, Faisalabad-38040, Pakistan. \\ *Corresponding author's e-mail: faizahmad1980@gmail.com
}

\begin{abstract}
Thermotoga maritima derived xylanases are important industrial enzymes owing to extensive thermotolerance and stability under broad $\mathrm{pH}$ range This study presents in-silico characterization of Thermotoga maritima derived xylanase A and xylanase $\mathrm{B}$ through homology modeling and molecular docking approaches. Analyses of primary and secondary structures revealed net negative charges on both xylanase A and xylanase B. Modular structure of xylanase A exhibited five significant domains including two CBM49, one GlycoHydro10 and two DUF1083. Whereas, structure of xylanase B contained one significant domain i.e. Glyco hydro 10. The amino acid residues Leu-54, Arg-85, Leu-66, Phe-68, Asn-81 and Ile-52 of CBM49 have shown interaction with xylan during docking analyses. Within second CBM49 domain, Tyr-96, Trp-47 and Arg-19 have shown interaction with xylan. No residue of GlycoHydro10 was found to interact with xylan. Whereas, Asn-55, Arg-83, Arg-60 and ILe-118 of DUF1083 interacted with xylan. The Phe-170, Trp-156, Glu-15, Ser-157 and Lys-17 of DUF-1083 domain of xylanase A also showed interaction with xylan. The Glu-15 and Phe-170 of xylanase B showed H-bonding while Lys-565 showed electrostatic interaction with xylan. Comparative phylogenetic analysis of xylanases from Thermotoga genus, showed the complex evolutionary lineage of multimeric xylanase A protein. The findings of this study will lead us towards functional insights of xylanases from Thermotoga maritima and will help the researchers in xylanase enzyme engineering through rational design for developing highly efficient industrial enzymes.
\end{abstract}

Keywords: Xylanases, Thermotoga maritima, in-silico analyses, Homology modeling, molecular docking, substrate interaction

\section{INTRODUCTION}

Xylanases are ubiquitous enzymes produced by prokaryotes as well as eukaryotes (Meeta and Anil, 2013). Xylanses are involved in the hydrolysis of xylan which an integral constituent of hemicellulosic cell wall (Kellete et al., 1990; Black et al., 1994). Xylan has great potential as renewable carbon and energy source through conversion to biofuels. Xylan is a hemicellulosic sub-component of phytobiomass that accounts for roughly $1 / 3^{\text {rd }}$ of all the renewable organic carbon globally. Therefore, development of inexpensive technologies where hemicellulose can be used as primary substrate is essential (Alvira et al., 2010). The xylanases of Thermotoga maritima are thermostable enzymes metabolizing a repertoire of carbohydrates including cellulose, hemicellulose, pectin, glucose, sucrose, xylan and many others (Nelson et al., 1999). Recently, xylanases have appealed substantial research interest owing to impending applications in paper \& pulp industry, food \& feed industry and fermentation industry (Kumasaka et al., 2005; Verma and
Satyanarayana, 2012). Xylanases hydrolyze xylans to yield mainly xylobiose as end product. Therefore, these enzymes are being employed in cost effective xylobiose production through xylan hydrolysis at commercial scale (Huang et al., 2019). In paper and pulp industry, xylanases are replacing chlorine for pulp bleaching as hydrolysis of xylan removes lignin from plant-based pulp. Similarly, xylanases have potential applications in enzyme-driven processes like clarification of juices, improvement in beer consistency, enhancing digestibility of animal feed stock and above all conversion of lignocellulosic biomass and agro-wastes to fermentation end-products (Gessesse and Gashe, 1997).

Xylanases have significance for industrial applications by virtue of their stability at high temperature and alkaline conditions (Kumasaka et al., 2005). The major advantage of using thermostable enzymes in industry is the enhanced rate of reaction at high temperature. For instance, the rate of reaction is known to be doubled by every $10^{\circ} \mathrm{C}$ rise in temperature, which in turn decreases the amount of enzyme 
needed, subsequently decreasing the cost of production (Zhou et al., 2011).

For the proper exploitation of these enzymes on industrial scale, detailed molecular characterization is essential. Although the protein structural and functional features can only be determined precisely by sophisticated experimental analyses (e.g. x-ray crystallography, NMR spectroscopy etc.), nonetheless; these analyses are very expensive, labor intensive and require larger amounts of purified sample as well as sound technical expertise (Ul Qamar and Khan, 2017). Computational tools are frequently available and are used for protein characterization and sequence analyses. These are cost effective and provide information nearly exact to be obtained via aforementioned experimental methods. Structural, structure-function relationship, interaction with substrate and other physico-chemical properties can be better explained using computational tools. However, comprehensive knowledge of structural biology and cuttingedge softwares are prime prerequisites for protein modeling (Sehar et al., 2013a).

In the present study, Xylanase A and Xylanase B of $T$. maritima were characterized in an in-silico study. Xylanases were characterized based on deduced 3D structures, physicochemical properties and phylogenetic relationships. Later, the xylanase-xylan interactions were determined using molecular docking. The findings of this study will lead towards functional insights of xylanases from Thermotoga maritima and will help the researchers in xylanase engineering through rational design for future exploitation in industrial and environmental applications.

\section{METHODOLOGY}

Protein sequence analyses: Amino acids sequences of xylanase A and xylanase B were retrieved from Uniprot (Bairoch et al., 2005) with accession numbers Q60037 and Q9WXS5, respectively. Primary sequence analyses of xylanases were performed using structure analyses tools available at ExPASy (Gasteiger et al., 2005). The GRAVY index was determined using ProtParam tool available at ExPASy. Secondary structure analyses were performed using PSIPRED server (McGuffin et al., 2000) and conserved domains were analysed using Pfam (Finn et al., 2016).

Construction of 3D structures and their analyses: The 3D structure of xylanase B was available in PDB (ID:1VBR) while $3 \mathrm{D}$ structures of xylanase $\mathrm{A}$ domains were predicted using homology modeling approach as described previously (Sehar et al., 2013b). Domain based structure modelling was performed because xylanase A exhibited exceptionally unique structure which could not be modeled using conventional protocols, and no suitable template was available for complete protein homology modeling. Domain wise multiple sequence alignment of xylanase A was performed by ClustalX2 (Ul Qamar and Khan, 2017)
Modeller 9.11 was employed for homology modelling and selection of template was the first step for homology modelling. For template selection, PSI-BLAST was run against PDB and 3D structure of templates were acquired from protein data bank (PDB). The template having at least $25 \%$ sequence identity and $60 \%$ query coverage was selected as per selection criteria (Fischer et al., 2001). After template selection, the alignment between template and query sequence was performed using Align2D script of Modeller 9.11. Followed by the construction of 3D structure of different domains of xylanase A using ModellingScript.

Five (5) models for each domain were constructed and one of each with best quality (having higher values on verify 3D, quality factor, Ramachandran plot) was selected. The predicted 3D structure was subjected to MolProbity and NIH server (SAVES) for analyses. Through MolProbity Ramachandran values, poor rotamers, $\mathrm{C}_{\beta}$ deviations, bad bonds and bad angels of residues were computed (Chen $\mathrm{et} \mathrm{al}$., 2010). Various evaluating tools including PROCHECK (Laskowski et al., 1996), ERRAT (Colovos and Yeates, 1993) and Verify_3D (Eisenberg et al., 1997) were accessed by NIH server (SAVES). The model for each domain with best values for all parameters (having higher values on verify $3 \mathrm{D}$, quality factor, Ramachandran plot) was selected. Later on, 3D structure of template and query protein was superimposed by UCSF Chimera for verification.

Molecular docking studies: To study the enzyme-substrate interactions, both enzymes were subjected to molecular docking analyses using a pentamer of xylan as ligand by AutoDockVina (Gessesse and Gashe, 2011). AutoDockVina was used for flexible ligand-protein docking. All steps for docking were performed iteratively for each domain. The 3D structure of ligand (xylan pentamer) was retrieved from PubChem (https://pubchem.ncbi.nlm.nih.gov/). The dock sites within protein structure were found by submitting the 3D structure of query protein at Pocket-Finder server (http://www.modelling.leeds.ac.uk/pocketfinder/). Different grid boxes were drawn for different domains. For studying molecular interaction of xylan with CBM49 domain of xylanase A, grid box having coordinates; centre- $\mathrm{x}=95.497$, centre- $y=24.935$, centre- $z=31.206$, size_ $x=40$, size_y $=40$ and size $z z=40$ was set. To find molecular interaction of xylan with second CBM49 domain of xylanase A, grid box having coordinates; centre- $x=36.303$, centre- $y=25.102$, centre- $\mathrm{z}=8.919$, size $\_\mathrm{x}=40$, size $\_\mathrm{y}=40$ and size $\_\mathrm{z}=40$ was set. For molecular interaction of xylan with GlycoHydro10 domain of xylanase A, grid box having coordinates; centre- $x$ $=-50.736$, centre $-\mathrm{y}=-20.285$, centre- $\mathrm{z}=-94.894$, size $\mathrm{x}=$ 60 , size $\_y=54$ and size $z z=40$ was set. To determine molecular interaction of xylan with DUF1083 domain of xylanase A, grid box having coordinates; centre- $x=32.748$, centre- $y=62.193$, centre- $z=21.419$, size_x $=40$, size_y $=40$ and size_z $=40$ was set. For studying molecular interaction of xylan with second DUF1083 domain of xylanase A, grid box 
having coordinates; centre-x $=27.353$, centre-y $=43.946$, centre- $z=16.382$, size_ $\mathrm{x}=40$, size_y $=40$ and size_ $\mathrm{z}=40$ was set. To determine interaction of xylan with xylanase $\mathrm{B}$, a grid box having coordinates; centre- $\mathrm{x}=22.644$, centre- $\mathrm{y}=$ 14.977 , centre-z $=18.992$, size_x $=40$, size_y $=40$ and size_z = 40 was set. Polar hydrogen molecules were added to all protein molecules and then docking analysis was carried out. Phylogenetic analysis: The evolutionary relationship of xylanase A and B from different bacteria was determined by reconstructing a phylogenetic tree using MEGA 7.0 (Hedges et al., 2015). Neighbour-joining method was used to infer the evolutionary history, while the Poisson correction method was used to figure out the evolutionary distances and their unit was the number of amino acid substitutions per site.

\section{RESULTS AND DISCUSSION}

Stability and solubility analyses: Here we have described structure prediction, important domains of xylanase A, molecular docking and phylogenetic analyses of xylanase A and xylanase B from T. maritima. We constructed 3D structure of xylanase A domains for the first time, refined and submitted to PDB (Table 1). According to our findings Xylanase A consisted of 273 residues. Among them, 154 residues showed negative while 119 residues appeared to have positive charges on them. On the other hand, xylanase B was relatively smaller molecule with a total of 96 residues with 52 negatively charged and 44 positively charged residues. Hence, it is concluded that both enzymes carry a net negative charge. Previously a thermostable family XynB (10 Endo-Xylanases) from $T$. maritima was characterized that cuts p-Nitrophenyl- $\beta$-D-xyloside (Zhengqiang et al., 2001). High affinity binding was observed in thermostable xylanase A with both insoluble xylan and insoluble cellulose (Boraston et al., 2001).

In the current study, physico-chemical properties like isoelectric point, instability index, GRAVY, amino acid and atomic compositions of the said enzyme were also determined. Summary of all such characteristics is given in Table 1. The instability indices of xylanase A \& B were found to be 29.79 and 38.41 , respectively. The stability of a query protein is estimated by its instability index. A protein with

Table 1. Physico-Chemical Parameters of Xylanase A and Xylanase B (Determined by ProtParam).

\begin{tabular}{|c|c|c|c|c|c|c|}
\hline Parameters & \multicolumn{3}{|c|}{ Xylanase A } & \multicolumn{3}{|c|}{ Xylanase B } \\
\hline Mol. Weight & \multicolumn{3}{|c|}{ 119643.2 Dalton } & \multicolumn{3}{|c|}{ 40674.5 Dalton } \\
\hline No. of amino acids & \multicolumn{3}{|c|}{1059} & \multicolumn{3}{|c|}{347} \\
\hline Theoretical pI & \multicolumn{3}{|c|}{5.02} & \multicolumn{3}{|c|}{5.64} \\
\hline Instability index (II) & \multicolumn{3}{|c|}{29.79 (stable) } & \multicolumn{3}{|c|}{38.41 (stable) } \\
\hline No. of Negatively Charged Residues (Asp + Glu) & \multicolumn{3}{|c|}{154} & \multicolumn{3}{|c|}{52} \\
\hline No. of Positively Charged Residues (Arg + Lys) & \multicolumn{3}{|c|}{119} & \multicolumn{3}{|c|}{44} \\
\hline \multirow[t]{5}{*}{ Atomic Composition } & Carbon & & 5407 & Carbon & & 1867 \\
\hline & Hydrogen & & 8340 & Hydrogen & & 2836 \\
\hline & Nitrogen & & 1386 & Nitrogen & & 474 \\
\hline & Oxygen & & 1638 & Oxygen & & 525 \\
\hline & Sulfur & & 21 & Sulfur & & 11 \\
\hline \multirow{22}{*}{ Amino Acid Composition } & Ala (A) & 67 & $6.3 \%$ & $\mathrm{Ala}(\mathrm{A})$ & 20 & $5.8 \%$ \\
\hline & $\operatorname{Arg}(\mathrm{R})$ & 34 & $3.2 \%$ & $\operatorname{Arg}(\mathrm{R})$ & 15 & $4.3 \%$ \\
\hline & $\operatorname{Asn}(\mathrm{N})$ & 54 & $5.1 \%$ & $\operatorname{Asn}(\mathrm{N})$ & 20 & $5.8 \%$ \\
\hline & Asp (D) & 73 & $6.9 \%$ & Asp (D) & 18 & $5.2 \%$ \\
\hline & Cys (C) & 1 & $0.1 \%$ & Cys (C) & 4 & $1.2 \%$ \\
\hline & Gln $(Q)$ & 36 & $3.4 \%$ & Gln (Q) & 7 & $2.0 \%$ \\
\hline & Glu (E) & 81 & $7.6 \%$ & Glu (E) & 34 & $9.8 \%$ \\
\hline & Gly (G) & 69 & $6.5 \%$ & Gly (G) & 18 & $5.2 \%$ \\
\hline & His $(\mathrm{H})$ & 12 & $1.1 \%$ & His $(\mathrm{H})$ & 8 & $2.3 \%$ \\
\hline & Ile (I) & 74 & $7.0 \%$ & Ile (I) & 28 & $8.1 \%$ \\
\hline & Leu (L) & 69 & $6.5 \%$ & Leu (L) & 27 & $7.8 \%$ \\
\hline & Lys (K) & 85 & $8.0 \%$ & Lys (K) & 29 & $8.4 \%$ \\
\hline & Met (M) & 20 & $1.9 \%$ & Met (M) & 7 & $2.0 \%$ \\
\hline & Phe $(\mathrm{F})$ & 45 & $4.2 \%$ & Phe $(F)$ & 18 & $5.2 \%$ \\
\hline & Pro (P) & 46 & $4.3 \%$ & Pro (P) & 14 & $4.0 \%$ \\
\hline & $\operatorname{Ser}(\mathrm{S})$ & 68 & $6.4 \%$ & $\operatorname{Ser}(S)$ & 16 & $4.6 \%$ \\
\hline & Thr (T) & 68 & $6.4 \%$ & Thr (T) & 11 & $3.2 \%$ \\
\hline & $\operatorname{Trp}(\mathrm{W})$ & 26 & $2.5 \%$ & $\operatorname{Trp}(\mathrm{W})$ & 10 & $2.9 \%$ \\
\hline & Tyr (Y) & 44 & $4.2 \%$ & Tyr (Y) & 19 & $5.5 \%$ \\
\hline & Val (V) & 87 & $8.2 \%$ & Val (V) & 24 & $6.9 \%$ \\
\hline & Pyl (O) & 0 & $0.0 \%$ & Pyl (O) & 0 & $0.0 \%$ \\
\hline & $\operatorname{Sec}(\mathrm{U})$ & 0 & $0.0 \%$ & $\operatorname{Sec}(\mathrm{U})$ & 0 & $0.0 \%$ \\
\hline Aliphatic Index & \multicolumn{3}{|c|}{82.81} & \multicolumn{3}{|c|}{87.64} \\
\hline Grand average of Hydropathicity (GRAVY) & \multicolumn{3}{|c|}{-0.390} & \multicolumn{3}{|c|}{-0.367} \\
\hline
\end{tabular}


instability index less than 40 is predicted to be stable and vice versa (Guruprasad et al., 1990). The present study revealed that xylanase $\mathrm{A}$ is more stable with instability index (29.79) than that of xylanase B with instability index (38.41). Second physiochemical parameter determined in present study was aliphatic index. The aliphatic index is termed as the relative volume of a protein molecule occupied by aliphatic side chains including alanine, valine, isoleucine and leucine. Aliphatic indices for xylanese A \& B were found to be 82.81 and 87.64, respectively. High aliphatic index is an indication of thermal stability of globular proteins (Ikai, 1980). Hence, it is concluded that both the xylanase molecules under study are thermostable.

The Grand average of hydropathy (GRAVY) value for a protein molecule is computed by dividing sum of hydropathy values of all residues by total number of residues in that sequence. It helps in determining hydrophilic or hydrophobic nature of a protein molecule. Hydrophilic nature of protein molecules was indicated by negative GRAVY value (Kyte and Doolittle, 1982). GRAVY value for xylanase A was 0.390 and for xylanase B was -0.367 which showed that both molecules were charge polarized and capable of establishing hydrogen bonds due to which their interaction with water and other polar solvents was expected to be favourable thermodynamically. Hence, this study revealed that both the enzymes under study were thermostable as well as hydrophilic in nature.

Secondary structure and domain analyses: The secondary structures of xylanase A and B were analyzed which exhibited $4.2 \% \alpha$ helices, $32.86 \% \quad \beta$ sheets and $62.94 \%$ loops in xylanase A while xylanase B contained 35\% $\alpha$ helices, $11 \% \beta$ sheets and 54\% loops. Domain analysis revealed that both enzymes possess multidomain structures which is a direct clue of a complex evolutionary track of said enzymes. Xylanase A contained five significant and two insignificant domains. Significant domains included two CBM49, one GlycoHydro10 and two DUF1083 while insignificant domains were DUF2374 and Laminin G3. It has been described that domains are sequences and structures of proteins that remained conserved during the course of evolution. Such a multi-domain structure of xylanase A reflects that the enzyme may have role in diverse metabolic pathways other than xylan degradation (Teng et al., 2014). On the other hand, xylanase B showed one significant and one insignificant domain including GlycoHydro10 and AAA18, respectively (Table $2 \mathrm{a}, 2 \mathrm{~b}$ ). The multiple sequence alignment of T. maritima xylanases showed their association with other members of Thermotoga genus, particularly xylanase A which has shown a close resemblance with Thermotoga sp. EMP, while xylanase B showed close resemblance with $T$. neapolitana (Yang and Han, 2018). Some most recent studies also revealed that xylanases with multidomain structures are more diverse in functions and more resilient to extreme conditions especially temperatures (Teo et al., 2019). Similarly, Nakamichi et al. (2019) also characterized a multidomain Xlyanase from a fungus Talaromyces cellulolyticus and found it to be bifunctional.

Homology modeling and molecular docking: The 3D structure of xylanase B was retrieved from PDB (1VBR) while structures of various domains of xylanase were predicted through homology modelling using Modeller 9.11. The criterion of template selection was based on sequence identity greater than $25 \%$ and lower E-value using PSIBLAST. For $1^{\text {st }}$ CBM49 domain, $2 \mathrm{~W} 5 \mathrm{~F}$ was selected as a template with $28 \%$ identity; For $2^{\text {nd }}$ CBM49 domain, $1 \mathrm{H} 6 \mathrm{X}$ was selected as a template with $33 \%$ identity; for GlycoHydro10 domain, 1N82 was selected as template with $46 \%$ identity; and for both DUF83 domains, 1I82 was used as a template with $26 \%$ and $100 \%$ identity, respectively. The best quality model is selected out of 5 for each domain of xylanase A (Fig. 1). Several methods including Ramachandran plot, Verify3D and ERRAT available at NIH server (SAVES) were used to check the consistency of predicted 3D structures (Table 3). Poor rotamers, $\mathrm{C}_{\beta}$ deviations, bad bonds and bad angels were determined through MolProbity (Table 4). All final models passed the

Table 2a. Domain analysis of Xylanase A (Determined by Pfam).

\begin{tabular}{lllll}
\hline Domains & Description & Amino acid Position & PMDB Ids & Signifiance \\
\hline CBM49 $\left(1^{\text {st }}\right)$ & Carbohydrate binding domain & $50-184$ & PM0082205 & Significant Pfam-A match \\
CBM49 $\left(2^{\text {nd }}\right)$ & Carbohydrate binding domain & $203-340$ & PM0082206 & Significant Pfam-A match \\
GlycoHydro10 & Glycosyl hydrolase family 10 & $369-693$ & PM0082207 & Significant Pfam-A match \\
DUF1083 $\left(1^{\text {st }}\right)$ & Domain of unknown Function & $715-869$ & PM0082208 & Significant Pfam-A match \\
DUF1083 $\left(2^{\text {nd }}\right)$ & Domain of unknown Function & $882-1059$ & PM0082209 & Significant Pfam-A match \\
DUF2374 & Protein of unknown function & $14-41$ & & Insignificant Pfam-A match \\
Laminin G3 & Concanavalin A-like & $238-367$ & & Insignificant Pfam-A match \\
& lectin/glucanases superfamily & & \\
\hline
\end{tabular}

Table 2b. Domain analysis of Xylanase B (Determined by Pfam).

\begin{tabular}{llll}
\hline Domains & Description & Amino acid Position & Significance \\
\hline GlycoHydro10 & Glycosyl hydrolase family 10 & $24-339$ & Significant Pfam-A match \\
AAA 18 & AAA domain & $24-113$ & Insignificant Pfam-A match \\
\hline
\end{tabular}


quality filtering tests. The 3D structures were furthermore validated by superimposition of predicted structures with their respective templates. UCSF Chimera's structure comparison command for superimposition was used for this purpose (Fig. 2 ), and it was assured that predicted models are accurate. Xylan binds to xylanases to change its structure due to hydrolysis and increases its activity.

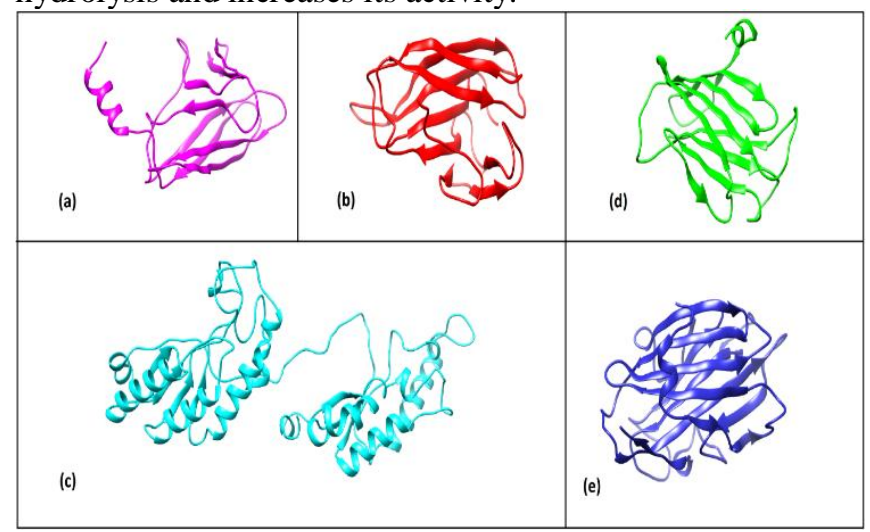

Figure 1.Models of Xylanase A domains, visualized by UCSF Chimera. (a) $1^{\text {st }}$ CBM49 domain (b) $2^{\text {nd }}$ CBM49 domain (C) GlycoHydro10 domain (d) $1^{\text {st }}$ DUF83 domain (e) $2^{\text {nd }}$ DUF83 domain.
The interactions do not cause inactivation of xylanases, so the hydrolysis rate is increased (Kaya et al., 2000). When ligand binds to receptor (enzyme) molecule, it alters the function of enzyme. The binding affinity can be characterized by the interaction of most ligands with their binding sites on receptors. High-affinity ligand binding is characterized by greater intermolecular force between ligand and receptors and vice versa (Eldridge et al., 1997).

After docking, interaction analyses were performed. The amino acid residues Leu-54, Arg-85, Leu-66, Phe-68, Asn-81 and Ile-52 of CBM49's domain 1 have shown interaction with xylan. Among them, The Asn-81, Ile-52, Leu-54 and Arg-85 showed H-bonding with the substrate. For second CBM49 domain, Tyr-96, Trp-47 and Arg-19 showed interaction with xylan. No residue of GlycoHydro10 was observed to bind with xylan. For DUF1083, Phe-120, Asn-55, Arg-83, Arg-60 and ILe-118 of DUF1083 interacted with xylan. Phe-120, Asn-55 and Arg-83 showed hydrogen bonding, while Arg-60 and Ile-118 showed electrostatic interactions. In xylanase A fifth domain, Phe-170, Trp-156, Glu-15, Ser-157 and Lys-17 showed interaction with xylan. In case of xylanase B, Glu-15 and Phe-170 showed H-bonding, while Lys-565 showed electrostatic interactions with xylan (Fig. 3).

Table 3. NIH Server (SAVES) analyses of template and models of the Xylanase A predicted by MODELLER. NIH Server (SAVES) evaluates the best selected models by using tools like ERRAT, Verify_3D and PROCHECK.

\begin{tabular}{lccc}
\hline Models & Verify3D & ERRAT Quality Factor & PROCHECK Ramachandran \\
\hline 2WYS (Template) & $97.07 \%$ & 97.811 & $89.0 \%$ \\
Xylanase A (1 ${ }^{\text {st }}$ CBM49) & $71.32 \%$ & 30.952 & $89.8 \%$ \\
1H6X (Template) & $90.63 \%$ & 84.768 & $86.8 \%$ \\
Xylanase A (2 ${ }^{\text {nd }}$ CBM49) & $99.28 \%$ & 31.538 & $91.7 \%$ \\
1N82 (Template) & $89.98 \%$ & 98.148 & $91.1 \%$ \\
Xylanase A (GlycoHydro10) & $85.09 \%$ & 59.105 & $91.1 \%$ \\
1I82 (Template) & $97.89 \%$ & 86.188 & $88.0 \%$ \\
Xylanase A (1 ${ }^{\text {st }}$ DUF1083) & $74.02 \%$ & 44.915 & $88.2 \%$ \\
1I82 (Template) & $97.89 \%$ & 86.188 & $88.0 \%$ \\
Xylanase A (2 & $91.01 \%$ & 88.757 & $94.2 \%$ \\
\hline
\end{tabular}

Table 4. MolProbity analyses of template and models of Xylanase A predicted by MODELLER.

\begin{tabular}{|c|c|c|c|c|c|c|}
\hline Models & $\begin{array}{c}\text { Poor } \\
\text { Rotamers }\end{array}$ & $\begin{array}{l}\text { Ramachandr } \\
\text { an Outliers }\end{array}$ & $\begin{array}{l}\text { Ramachandr } \\
\text { an Favored }\end{array}$ & $\begin{array}{c}C \beta \text { deviations } \\
>0.25 \AA\end{array}$ & $\begin{array}{l}\text { Residues with } \\
\text { bad bonds }\end{array}$ & $\begin{array}{l}\text { Residues with } \\
\text { bad angles }\end{array}$ \\
\hline 2WYS (Template) & $2.32 \%$ & $0.79 \%$ & $95.47 \%$ & $0.31 \%$ & $0.02 \%$ & $0.06 \%$ \\
\hline Xylanase A ( $1^{\text {st }}$ CBM49) & $2.56 \%$ & $1.50 \%$ & $91.73 \%$ & $0.00 \%$ & $0.00 \%$ & $0.00 \%$ \\
\hline 1H6X (Template) & $0.78 \%$ & $0.00 \%$ & $98.09 \%$ & $0.00 \%$ & $0.00 \%$ & $0.00 \%$ \\
\hline Xylanase A ( $2^{\text {nd }}$ CBM49) & $2.54 \%$ & $1.47 \%$ & $97.79 \%$ & $0.00 \%$ & $0.00 \%$ & $0.00 \%$ \\
\hline 1N82 (Template) & $1.59 \%$ & $0.00 \%$ & $98.32 \%$ & $0.15 \%$ & $0.00 \%$ & $0.06 \%$ \\
\hline Xylanase A (GlycoHydro10) & $2.45 \%$ & $1.88 \%$ & $95.30 \%$ & $0.65 \%$ & $0.00 \%$ & $0.81 \%$ \\
\hline 1I82 (Template) & $0.63 \%$ & $0.00 \%$ & $96.26 \%$ & $0.00 \%$ & $0.00 \%$ & $0.32 \%$ \\
\hline XylanaseA ( $1^{\text {st }}$ DUF 1083$)$ & $0.89 \%$ & $2.42 \%$ & $92.74 \%$ & $0.84 \%$ & $0.00 \%$ & $0.16 \%$ \\
\hline 1I82 (Template) & $0.63 \%$ & $0.00 \%$ & $96.26 \%$ & $0.00 \%$ & $0.00 \%$ & $0.32 \%$ \\
\hline Xylanase A ( $\left(2^{\text {nd }}\right.$ DUF 1083) & $2.60 \%$ & $0.00 \%$ & $98.29 \%$ & $0.00 \%$ & $0.00 \%$ & $0.00 \%$ \\
\hline
\end{tabular}




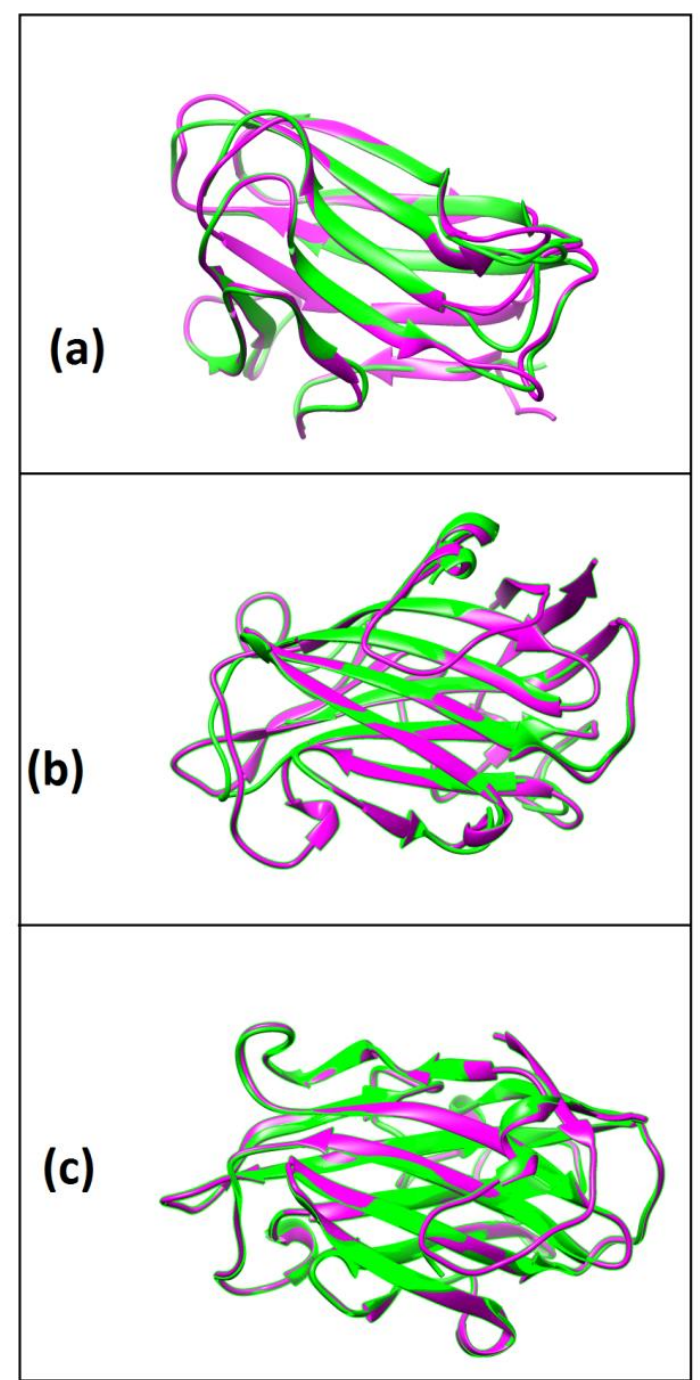

Figure 2. Superimposition of models with their entire template, using UCSF Chimera. (a) Superimposition of CBM49 domain (Green) with 1H6X template (Magenta), (b) Superimposition of DUF83 domain (Green) with 1 I82 template (Magenta), (c) Superimposition of DUF83 domain (Green) with 1182 template (Magenta).

Binding specificity of CBM9-2, a domain of xylanase A, has already been reported. Binding of CBM9-2 to barley xyloglucan and $\beta$-glucan and weakly to wheat arabinoxylan and soluble birchwood xylan endorsed the findings of present study. The receptors for hydroxyethylcellulose, dextran arabinan, arabinogalactan, CM-cellulose, pecticgalactan, starch, laminarin, or locust bean gum are not present. The direct interaction of CBM9-2 with reducing end of polysaccharides is the most novel property (Yang and Han, 2018; Teo et al., 2019; Tassa et al., 2009). Xylanase B could bind to both insoluble xylan and Avicel even though it had no activity on Avicel. Several solvent-exposed aromatic rings mediate the binding by hydrogen bonding and stacking interactions with sugar in polysaccharides (Huang et al., 2019; Nakamichi et al., 2019).

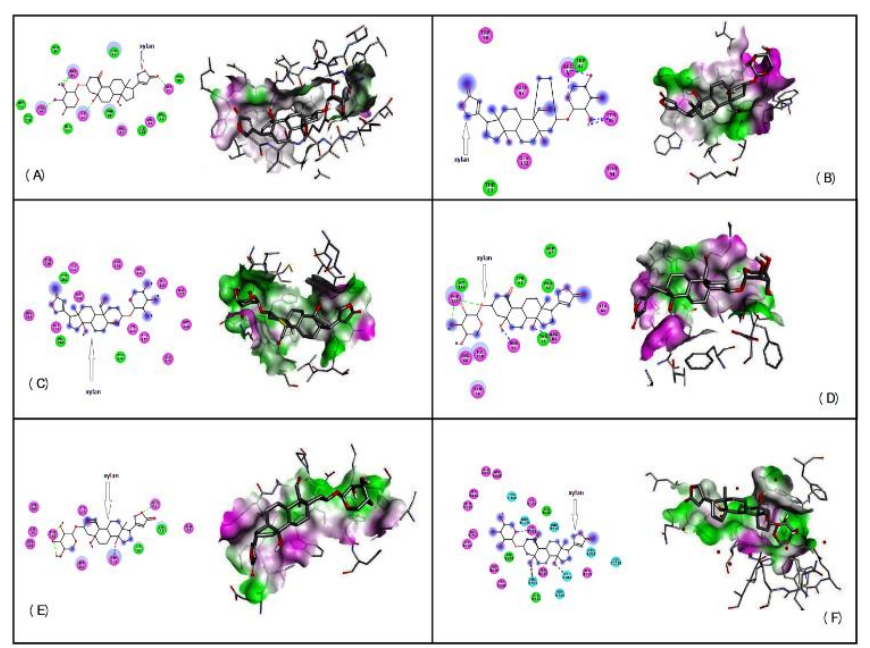

Figure 3. Residues of all domains of Xylanase $A$ and Xylanase B interacting with xylan are shown. (A) $1^{\text {st }}$ CBM49 domain interaction with xylan, (B) $2^{\text {nd }}$ CBM49 domain interaction with xylan, $(C)$ GlycoHydro10 domain interaction with xylan, (D) $1^{\text {st }}$ DUF83 domain interaction with xylan, (E) $2^{\text {nd }}$ DUF83 domain interaction with xylan, (F) GlycoHydro10 domain of xylanase B interaction with xylan.

Phylogenetic analyses: The xylanases have been sequenced from a variety of bacteria and sequences have been submitted to protein sequence databases. Evolutionary relationship among submitted sequences can be revealed by sequences alignment and subsequent phylogenetic analysis of xylanase A \& B from a variety of microorganisms. The phylogenetic analyses showed that xylanase A and B of T. maritima have diverse evolutionary relationship, not only within the genus Thermotoga but also with other microbial genera. Both xylanases were grouped in different clans showing their evolutionary divergence. We used neighbour-joining method to deduce evolutionary history of enzymes under study (Ul Qamar and Khan, 2017). Figure 4 shows optimal tree with a total of branch length $=5.27560916$. The percentage of replicate trees where the related taxa clustered together in the bootstrap test (1000 replicates) was marked next to the branches. The units of branch lengths in phylogenetic tree were same as those of the evolutionary distances used while inferring the phylogenetic tree. The scale bar corresponds to 0.1 -estimated amino acid substitution per sequence position. The analysis involved 26 amino acid sequences with elimination of gaps and missing data. Final dataset contains 


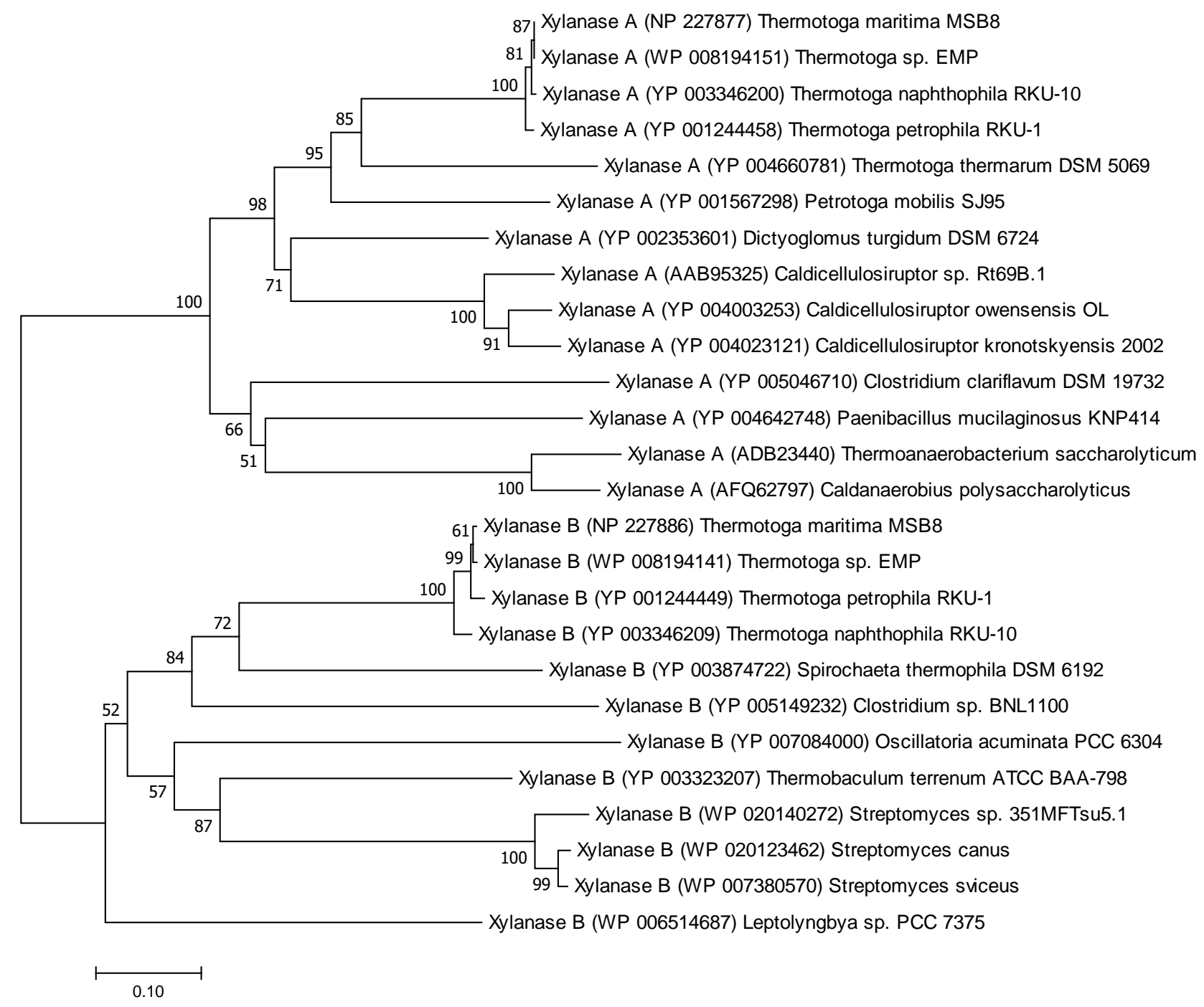

Figure 4. Phylogenetic tree based on microbial xylanase amino acid sequences.

total of 302 positions. MEGA7 software package was used to conduct evolutionary analyses (Sehar et al., 2013b). Xylanase amino acid sequences along with accession numbers (NCBI) from different microorganisms are represented in taxa. This further demonstrates that variety of physiochemical and thermodynamic characteristics of xylanases isolated from different groups of organisms also represents variation at protein sequence level.

Epilogue: This study leads us to better understanding of enzyme-substrate interaction in prokaryotes especially in extremophiles. It also lead us towards functional insights of xylanases from Thermotoga maritima and will help the researchers interested in xylanase enzyme engineering through rational design to develop highly efficient enzymes for future exploitation in industrial and environmental applications.

\section{REFERENCES}

Alvira, P., E. Tomás-Pejó, M. Ballesteros and M.J. Negro. 2010. Pretreatment technologies for an efficient bioethanol production process based on enzymatic hydrolysis: a review. Biores. Technol. 101:4851-4861.

Bairoch, A.M., R. Apweiler, C.H. Wu, W.C. Barker, B. Boeckmann, S.F. Rojas, E. Gasteiger, H. Huang, R. Lopez, M. Magrane and M.J. Martin. 2005. The universal protein resource (UniProt). Nucl. Acids Res. 33:D154D159.

Black, G.W., G.P. Hazlewood, G.P. Xue, C.G. Orpin and H.J. Gilbert. 1994. Xylanase B from Neocallimastix patriciarum contains a non-catalytic 455-residue linker 
sequence comprised of 57 repeats of an octapeptide. Biochem. J. 299:381-387.

Boraston, A.B., A.L. Creagh, M.M. Alam, J.M. Kormos, P. Tomme, C.A. Haynes, R.A. Warren and D.G. Kilburn. 2001. Binding specificity and thermodynamics of a family 9 carbohydrate-binding module from Thermotoga maritima xylanase 10A. Biochemistry 40:6240-6247.

Chen, V.B., W.B. Arendall, J.J. Headd, D.A. Keedy, R.M. Immormino, G.J. Kapral, L.W. Murray, J.S. Richardson and D.C. Richardson. 2010. MolProbity: all-atom structure validation for macromolecular crystallography. Acta Crystallogr. D Biol. Crystallogr. 66:12-21.

Colovos, C. and T.O. Yeates. 1993. Verification of protein structures: patterns of nonbonded atomic interactions. Prot. Sci. 2:1511-1519.

Eisenberg, D., R. Lüthy and J.U. Bowie. 1997. VERIFY3D: Assessment of protein models with three-dimensional profiles. Meth. Enzymol. 277:396-404.

Eldridge, M.D., C.W. Murray, T.R. Auton, G.V. Paolini and R.P. Mee. 1997. Empirical scoring functions: I. The development of a fast empirical scoring function to estimate the binding affinity of ligands in receptor complexes. J. Computer-aided Mol. Des. 11:425-445.

Finn, R.D., P, Coggill, R.Y. Eberhardt, S.R. Eddy, J. Mistry, A.L. Mitchell, S.C. Potter, M. Punta, M. Qureshi, A. Sangrador-Vegas and G.A. Salazar. 2016. The Pfam protein families database: towards a more sustainable future. Nucl. Acids Res. 44:D279-D285.

Fischer, D., A. Elofsson, L. Rychlewski, F. Pazos, A. Valencia, B. Rost, A.R. Ortiz and R.L. Dunbrack. 2001. CAFASP2: the second critical assessment of fully automated structure prediction methods. Proteins 45:171-183.

Gasteiger, E., C. Hoogland, A. Gattiker, S.E. Duvaud, M.R. Wilkins, R.D. Appel and A. Bairoch. 2005. Protein identification and analysis tools on the ExPASy server. In The proteomics protocols handbook Humana Press.

Gessesse, A. and B.A. Gashe. 1997. Production of alkaline xylanase by an alkaliphilic Bacillus sp. isolated from an alkalinesoda lake. J. Appl. Microbiol. 83:402-406.

Guruprasad, K., B.V. Reddy and M.W. Pandit. 1990. Correlation between stability of a protein and its dipeptide composition: a novel approach for predicting in vivo stability of a protein from its primary sequence. Prot. Eng. Des. Sel. 4:155-161.

Hedges, S.B., J. Marin, M. Suleski, M. Paymer and S. Kumar. 2015. Tree of life reveals clock-like speciation and diversification. Mol. Biol. Evol. 32:835-845.

Huang, Y., X. Zheng, B. Pilgaard, J. Holck, J. Muschiol, S. Li and L. Lange. 2019. Identif ication and characterization of GH11 xylanase and GH43 xylosidase from the chytridiomycetous fungus, Rhizophlyctis rosea. Appl. Microbiol. Biotechnol. 103:777-791.
Ikai, A. 1980. Thermostability and aliphatic index of globular proteins. J. Biochem. 88:1895-1898.

Kaya, F., J.A. Heitmann and T.W. Joyce. 2000. Influence of lignin and its degradation products on enzymatic hydrolysis of xylan. J. Biotechnol. 80:241-247.

Kellett, L.E., D.M. Poole, L.M. Ferreira, A.J. Durrant, G.P. Hazlewood and H.J. Gilbert. 1990. Xylanase B and an arabinofuranosidase from Pseudomonas fluorescens subsp. cellulosa contain identical cellulose-binding domains and are encoded by adjacent genes. Biochem. J. 272:369-376.

Kumasaka, T., T. Kaneko, C. Morokuma, R. Yatsunami, T. Sato, S. Nakamura and N. Tanaka. 2005. Structural basis of the substrate subsite and the highly thermal stability of xylanase 10B from Thermotoga maritima MSB8. PROTEINS: Struct. Funct. Bioinform. 61:999-1009.

Kyte, J. and R.F. Doolittle. 1982. A simple method for displaying the hydropathic character of a protein. J. Mol. Biol. 157:105-132.

Laskowski R.A., J.A. Rullmann, M.W. MacArthur, R. Kaptein and J.M. Thornton. 1996. AQUA and PROCHECK-NMR: programs for checking the quality of protein structures solved by NMR. J. Biomol. NMR 8:477-486.

McGuffin, L.J., K. Bryson and D.T. Jones. 2000. The PSIPRED protein structure prediction server. Bioinformatics 16:404-405.

Meeta, S. and K. Anil. 2013. Xylanases: an overview. Brit. Biotechnol. J. 3:1-28.

Nakamichi, Y., T. Fouquet, S. Ito, M. Watanabe, A. Matsushika and H. Inoue. 2019. Structural and functional characterization of a bifunctional GH30-7 xylanase B from the filamentous fungus Talaromyces cellulolyticus. J. Biol. Chem. 294:4065-4078.

Nelson, K.E., R.A. Clayton, S.R. Gill, M.L. Gwinn, R.J. Dodson, D.H. Haft, E.K. Hickey, J.D. Peterson, W.C. Nelson, K.A. Ketchum and L. McDonald. 1999. Evidence for lateral gene transfer between Archaea and bacteria from genome sequence of Thermotoga maritima. Nature 399:323-329.

Sehar, U., M.A. Mehmood, K. Hussain, S. Nawaz, S. Nadeem, M.H. Siddique, H. Nadeem, M. Gull, N. Ahmad, I. Sohail and S.S. Gill. 2013a. Domain wise docking analyses of the modular chitin binding protein CBP50 from Bacillus thuringiensis serovar konkukian S4. Bioinformation 9:901-907.

Sehar, U., M.A. Mehmood, S. Nawaz, K. Hussain, I. Sohail, M.R. Tabassum, S.S. Gill and A. Saqib. 2013b Three dimensional (3D) structure prediction and substrateprotein interaction study of the chitin binding protein CBP24 from B. thuringiensis. Bioinformation 9:725-729.

Tassa, C., J.L. Duffner, T.A. Lewis, R. Weissleder, S.L. SchreiberL, A.N. Koehler and S.Y. Shaw. 2009. Binding 
affinity and kinetic analysis of targeted small moleculemodified nanoparticles. Bioconj. Chem. 21:14-19.

Teng, Z., M. Guo, Q. Dai, C. Wang, J. Li and X. Liu. 2014. Computational prediction of protein function based on weighted mapping of domains and GO terms. BioMed Res. Int. 2014:1-9.

Teo, S.C., K.J. Liew, M.S. Shamsir, C.S. Chong, N.C. Bruce, K.G. Chan and K.M. Goh. 2019. Characterizing a HaloTolerant GH10 Xylanase from Roseithermus sacchariphilus Strain RA and Its CBM-Truncated Variant. Int. J. Mol. Sci. 2019; 20:2284.

Ul Qamar M.T. and M.S. Khan. 2017. A novel structural and functional insight into chloroplast-encoded central subunit of dark-operated protochlorophyllide oxidoreductase (DPOR) of plants. Pak. J. Agri. Sci. 54:395-406.
Verma, D. and T. Satyanarayana. 2012. Molecular approaches for ameliorating microbial xylanases. Biores. Technol. 117:360-367.

Yang, J. and Z. Han. 2018. Understanding the Positional Binding and Substrate Interaction of a Highly Thermostable GH10 Xylanase from Thermotoga maritima by Molecular Docking. Biomolecules 8:64. doi:10.3390/biom8030064

Zhengqiang, J., A. Kayashi, M.M. Ahsan, L. Lite, M. Kitaoka and K. Hayashi. 2001. Characterization of a thermostable family 10 endo-xylanase (XynB) from Thermotoga maritima that cleaves $\mathrm{p}$-nitrophenyl- $\beta$-D-xyloside. J. Biosc. Bioeng. 92:423-428.

Zhou, P., H. Zhu, Q. Yan, P. Katrolia and Z. Jiang. 2011. Purification and properties of a psychrotrophic Trichoderma sp. xylanase and its gene sequence. Appl. Biochem. Biotechnol. 164:944-956. 